\title{
Modulated speckle simulations based on the random-walk model
}

\author{
A. Lencina and P. Vaveliuk \\ Laboratório de Óptica Não Linear, Departamento de Física, Centro de Ciências Exatas e da Natureza, \\ Universidade Federal da Paraíba, Brasil
}

M. Tebaldi

Centro de Investigaciones Ópticas, Consejo Nacional de Investigaciones Científícas Técnicas, CIC, and Facultad Ingenieria, Universidad Nacional de la Plata, Argentina

N. Bolognini

Centro de Investigaciones Ópticas, Consejo Nacional de Investigaciones Científícas Técnicas, CIC, and Facultad Ciencias Exactas, Universidad Nacional de la Plata, Argentina

Received December 2, 2002

The random-walk model is employed to simulate modulated speckle patterns. We demonstrate that the geometrical image approximation fails to describe the modulated speckle pattern. A new approach to analyzing this phenomenon is proposed. The validity of the approximations employed is verified by comparison of the simulation with the experimental results. Speckle metrological applications and phase measurement techniques could be improved by taking advantage of this model. (C) 2003 Optical Society of America

OCIS codes: $\quad 070.2580,110.1220,110.2990,110.6150$.

Computational developments over the past few years offer new possibilities for the random-walk model. This model allows a physical magnitude to be described as a finite sum of random phasors and is usually utilized to describe several effects involved in the propagation of electromagnetic waves. ${ }^{1}$ As is well known, speckles are generated when multiple sources of interference scatter from a diffusing object illuminated by coherent light. ${ }^{2}$ Then the diffuser can be assumed to be an ensemble of scattering elements with any distribution of shape, position, and phase. In speckle applications a continuous model for the speckle field is used, thereby restricting the results to mean values. We believe that the random-walk model could help in understanding and lead to a further analysis of the phenomenon based on a point-by-point approach. In particular, we propose using the random-walk model to revise the analysis of the modulated speckle pattern that appears in several metrological applications.,4 The speckle simulation can be used to test the geometrical image approximation and to verify its validity. In particular, we find that the approximation fails to describe thoroughly the modulated speckle pattern. Therefore we propose a new approach to analyzing this phenomenon.

In the proposed experimental setup a laser beam of wavelength $\lambda$ impinges on the diffuser ( $x-y$ plane). An image of the diffuser is formed in the $X-Y$ plane by use of a lens of focal length $f$. A pupil mask with several identical apertures $a_{h}$ (where $h \equiv 1,2, \ldots, n$ ) is located immediately in front of the lens. There is no overlap between two different apertures belonging to the same pupil. Note that the line that joins a particular aperture center and the point that results from the intersection between the optical axis of the imaging system and the pupil mask plane forms an angle $\alpha_{h}$ with the horizontal axis ( $u$ axis). Also, the center of the aperture $a_{h}$ is located at a distance $d_{h}$ from the optical axis

0146-9592/03/191748-03\$15.00/0 of the system. $Z_{0}$ and $Z_{C}$ denote the distance from the diffuser to the lens and from the lens to the image plane, respectively. Let us introduce an experimental result to provide a basis for our theoretical approach. In Fig. 1(a) a magnified speckle pattern obtained in the $X-Y$ plane is shown. The image is generated by use of a double-aperture pupil with circular holes of diameter $D \equiv 4.8 \mathrm{~mm}$ and separated a distance $d \equiv 9.2 \mathrm{~mm}$. The parameters are $\lambda=514 \mathrm{~nm}, Z_{0} \equiv 138 \mathrm{~mm}$, and $Z_{C}=382 \mathrm{~mm}$.

Let us analyze theoretically the amplitude distribution in the image plane. The speckle image field
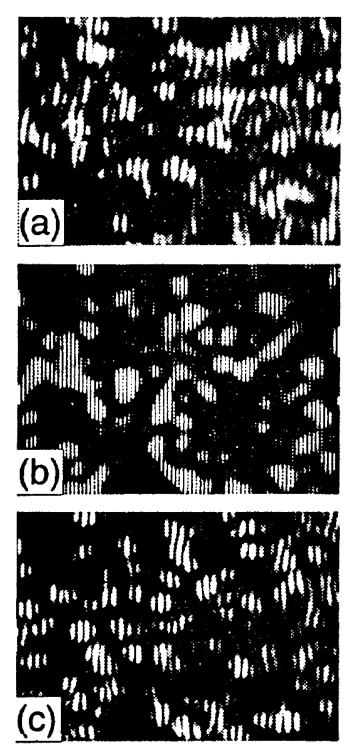

Fig. 1. (a) Experimental modulated speckle pattern. Theoretically modulated speckle pattern obtained by use of (b) geometrical image approximation and (c) local phase approximation. 
in the $X-Y$ plane obtained by use of the RayleighSommerfeld expression (see Ref. 5) is given by

$$
\begin{aligned}
& U_{i}(X, Y)=\iiint \int U_{0}(x, y) \frac{\exp (-j k R)}{\lambda R} P(u, v) \\
& \quad \times \exp \left[j \frac{k}{2 f}\left(u^{2}+v^{2}\right)\right] \frac{\exp \left(-j k R^{\prime}\right)}{\lambda R^{\prime}} \mathrm{d} x \mathrm{~d} y \mathrm{~d} u \mathrm{~d} v,
\end{aligned}
$$

where $k=2 \pi / \lambda, j=\sqrt{-1}, R^{2}=Z_{0}^{2}+(v-y)^{2}+(u-$ $x)^{2}$, and $R^{\prime 2}=Z_{C}^{2}+(v-Y)^{2}+(u-X)^{2}$.

To generalize, let us consider a multiple-aperture arrangement. In this case the pupil function in Eq. (1) consists of a sum over all the apertures; that is, $P(u, v)=-\sum_{h=1}^{n} a_{h}\left(u-d_{h} \cos \alpha_{h}, v-d_{h} \sin \alpha_{h}\right)$. Let us replace the explicit pupil function in Eq. (1) and introduce a variable change $u_{h}=u-d_{h} \cos \alpha_{h}$ and $v_{h}=v-d_{h} \sin \alpha_{h}$ for each aperture. Besides, by considering the well-known paraxial approximations $\left(Z_{0} \gg d_{h}, u_{h}, v_{h}\right.$ and $\left.Z_{C} \gg d_{h}, u_{h}, v_{h}\right)$, we get

$$
\begin{aligned}
& U_{i}(X, Y)=\frac{1}{\lambda^{2} Z_{0} Z_{C}} \sum_{h=1}^{n} \iiint \int U_{0}(x, y) a_{h}\left(u_{h}, v_{h}\right) \\
& \times \exp \left\{\frac{-j k}{2 Z_{C}}\left[\left(d_{h} \sin \alpha_{h}-Y\right)^{2}+\left(d_{h} \cos \alpha_{h}-X\right)^{2}\right]\right\} \\
& \quad \times \exp \left\{\frac{-j k}{2 Z_{0}}\left[\left(d_{h} \sin \alpha_{h}-y\right)^{2}+\left(d_{h} \cos \alpha_{h}-x\right)^{2}\right]\right\} \\
& \quad \times \exp \left\{j k\left[u_{h}\left(\frac{x}{Z_{0}}+\frac{X}{Z_{C}}\right)+v_{h}\left(\frac{y}{Z_{0}}+\frac{Y}{Z_{C}}\right)\right]\right\} \\
& \quad \times \exp \left\{j k\left[\frac{d_{h}^{2}}{2 f}-\left(Z_{0}+Z_{C}\right)\right]\right\} \mathrm{d} x \mathrm{~d} y \mathrm{~d} u_{h} \mathrm{~d} v_{h} .
\end{aligned}
$$

Note that Eq. (2) explicitly shows the coordinate system variable associated with each aperture. At this point it is important to analyze the square phase terms in Eq. (2). The term $\exp \left\{-j k\left[\left(d_{h} \sin \alpha_{h}-Y\right)^{2}+\right.\right.$ $\left.\left.\left(d_{h} \cos \alpha_{h}-X\right)^{2}\right] / 2 Z_{C}\right\}$ is relevant because it depends on the summation index. In a previous paper, when a single-aperture pupil was considered, this term went out of the integral and vanished if the intensity evaluation was done. The second square term to be analyzed is $\exp \left\{-j k\left[\left(d_{h} \sin \alpha_{h}-y\right)^{2}+\left(d_{h} \cos \alpha_{h}-\right.\right.\right.$ $\left.\left.x)^{2}\right]\right\} / 2 Z_{0}$. In previous studies, ${ }^{6}$ for instance, this term was disregarded because a geometrical image approximation was done. In this approach the light amplitude at coordinates $(X, Y)$ must consist of contributions from a tiny region of the object space, centered at the ideal geometrical object point. If within that tiny region the argument of $\exp \left[j k\left(x^{2}+y^{2}\right) / 2 Z_{0}\right]$ changes by no more than a fraction of a radian, then the approximation $x \cong X / M$ and $y \cong Y / M$, where $M$ is the image magnification, is used. The random-walk model is an adequate tool ${ }^{7}$ for obtaining an explicit expression of the amplitude field. In this model the speckle field $U_{0}(x, y)=\sum_{q}^{r} \equiv{ }_{1} U_{q}(x, y) \exp \left(j \phi_{q}\right)$ consists of a sum of $r$ discrete field amplitudes $U_{q}(x, y)$ with phase $\phi_{q}$, which contribute to the image speckle field formation. These discrete field components are small spots with random amplitude, phase, and position $\left(x_{q}, y_{q}\right)$.

At this point, to evaluate the image field, the random-walk model is introduced. In this evaluation the approximations concerning the second square term ${ }^{6}$ detailed above are taken into account. In this case, by neglecting the phase terms that go out of the integrals and sums, the amplitude field becomes

$$
\begin{aligned}
U_{i}(X, Y)= & \frac{1}{\lambda^{2} Z_{0} Z_{C}} \sum_{q=1}^{r} \sum_{h=1}^{n} U_{q h}(X, Y) \\
& \times \exp \left\{j\left[\phi_{q}+\phi_{h}(X, Y)\right]\right\},
\end{aligned}
$$

where

$$
\begin{aligned}
\phi_{h}(X, Y)= & \frac{-2 k d_{h}}{Z_{C}}\left(Y \sin \alpha_{h}+X \cos \alpha_{h}\right), \\
U_{q h}(X, Y)= & \iint \mathrm{d} x \mathrm{~d} y U_{q}(x, y) A_{h}(x, y ; X, Y), \\
A_{h}(x, y ; X, Y)= & \iint a_{h}\left(u_{h}, v_{h}\right) \\
& \times \exp \left\{j k \left[u_{h}\left(\frac{x}{Z_{0}}+\frac{Y}{Z_{C}}\right)\right.\right. \\
& \left.\left.+y_{h}\left(\frac{y}{Z_{0}}+\frac{Y}{Z_{C}}\right)\right]\right\} \mathrm{d} u_{h} \mathrm{~d} v_{h} .
\end{aligned}
$$

Let us evaluate the intensity. A double-aperture pupil system is considered. Both apertures are identical and are separated by a distance $d$ symmetrically with respect to the optical axis. The line joining the apertures is horizontal. Therefore the value of the angle $\alpha_{h}$ that corresponds to each aperture is $\alpha_{1} \equiv 0$ and $\alpha_{2} \equiv \pi$, respectively. In this case the intensity can be written as

$$
\begin{aligned}
I_{i}(X, Y)= & \frac{2}{\left(\lambda^{2} Z_{0} Z_{C}\right)^{2}}\left[1+\cos \left(\frac{2 k d X}{Z_{C}}\right)\right] \\
& \times\left[\sum_{q=1}^{r} U_{q}^{2}+2 \sum_{q=1}^{r-1} \sum_{s>q}^{r} U_{q} U_{s} \cos \left(\phi_{q}-\phi_{s}\right)\right] .
\end{aligned}
$$

This theoretical intensity pattern is depicted in Fig. 1(b). In this case the same parameters as in Fig. 1(a) are employed. In the random-walk model the speckle field in the diffuser plane originates from $r$ points, each one with associated amplitude, phase, and position. In this case the image area is $\approx 0.53 \mathrm{~mm}^{2}$, and the area of each speckle is $\approx 0.0025 \mathrm{~mm}^{2}$. Under this condition approximately 200 speckles are necessary to fulfill the corresponding region. Nevertheless, to ensure Gaussian behavior, we include 600 speckles in our simulations. In the model the speckles are uniformly and randomly distributed in the $x-y$ plane and have random amplitudes in the range $[0,1]$. Also, to ensure a fully developed speckle regime, the phases are assumed randomly and uniformly distributed in the range $[=\pi, \pi]$. It should be mentioned that the random values in the simulations are obtained by use of the Mathematica software package that employs the Marsaglia-Zaman subtract-with-borrow (SWB) generator. ${ }^{8}$

It should be noted that the fringe frequency that modulates the speckle pattern obtained by use of Eq. (7) [Fig. 1(b)] differs from the experimental case 
[Fig. 1(a)]. A simple geometrical analysis shows that the spatial frequency for a double-aperture system is $|\mathbf{K}|=k d / Z_{C}$. However, from Eq. (7) the spatial frequency is $\left|\mathbf{K}^{\prime}\right|=2 k d / Z_{C}$, which confirms the discrepancies between the results of Figs. 1(a) and 1(b). Also, it is observed that the fringe modulation phase in the theoretical pattern does not depend on the speckle position. This behavior does not coincide with the experimental result. These discrepancies have motivated us to make a further analysis. Let us reinterpret the approximations in the second square term $\exp \left\{-j k\left[\left(d_{h} \sin \alpha_{h}-y\right)^{2}+\left(d_{h} \cos \alpha_{h}-x\right)^{2}\right] / 2 Z_{0}\right\}$, where now the geometrical image approximation is removed.

We have an explicit expression for the field $U_{0}(x, y)=$ $\sum_{q=1}^{r} U_{q}(x, y) \exp \left(j \phi_{q}\right)$. This field is discrete and implies evaluation of the integration in each spot [see Eq. (2)]. Besides, because of its small size, it is valid to fix a local phase value by assuming a low-phase variation in it. This local phase implies replacing $(x, y)$ by $\left(x_{q}, y_{q}\right)$ in the square term mentioned.

The intensity applied to an identical system, as in Eq. (7), under the conditions detailed above and neglecting phase terms that go out of the integrals and sums yields

$$
\begin{aligned}
I_{i}(X, Y) \frac{\left(\lambda^{2} d_{0} d_{i}\right)^{2}}{2}= & \sum_{q=1}^{r} U_{q}^{2}\left\{1+\cos \left[k d\left(\frac{x_{q}}{Z_{0}}+\frac{X}{Z_{C}}\right)\right]\right\} \\
& +\sum_{q=1}^{r-1} \sum_{s>q}^{r} U_{q} U_{s} \cos \left[\phi_{q}-\phi_{s}\right. \\
& \left.+\frac{k}{2 Z_{0}}\left(y_{q}^{2}-y_{s}^{2}+x_{q}^{2}-x_{s}^{2}\right)\right] \\
& \times\left\{\cos \left[\frac{k d}{2 Z_{0}}\left(x_{q}-x_{s}\right)\right]\right. \\
& \left.+\cos \left[k d\left(\frac{x_{q}+x_{s}}{2 Z_{0}}+\frac{X}{Z_{C}}\right)\right]\right\}
\end{aligned}
$$

By use of Eq. (8) a new theoretical intensity pattern is obtained. This result is depicted in Fig. 1(c). The same parameters as in Fig. 1(b) are used. It is apparent that Figs. 1(a) and 1(c) exhibit similar features. Note that the fringe frequency coincides in both figures and a local phase behavior as in the experimental case is observed in Fig. 1(c).

In this study we demonstrate that by use of the random-walk model and with a new approach for the optical model, it is possible to simulate modulated speckle patterns whose behavior coincides with the experimental results. The analysis is general and could be applied to more complex aperture pupils. In Ref. 9 a peculiar type of speckle pattern was reported. These speckles present a clustering appearance consisting of many chains of speckle grains. This feature is attributed to the circular symmetry of the ring-slit aperture employed. The dynamic of clustering could be analyzed by applying the proposed model to an optical system that gradually increases the circular symmetry of the pupil apertures. To this end, a ring-slit pupil aperture can be synthesized by circular aperture pairs placed along a diameter of the ring. Also, the versatility of the random-walk model allows for the simulation of speckle patterns with different statistical properties in terms of the statistical properties of the diffuser field.

This approach, which yields a more precise description of the speckles' local behavior, could be useful in controlling the diffuser features, for instance, its statistics (fully developed or Gaussian) and surface roughness. Note that in the speckle photography technique a Fourier plane analysis of the specklegram is done. This approach is basically followed in Ref. 10 and does not allow local phase changes due to the presence of complex phase objects in the pupil plane to be determined. ${ }^{10}$ Nevertheless, the information needed to determine these local changes is present in the specklegram. In this study a theoretical model that shows the local behavior of the modulated fringe distribution has been outlined. Let us introduce a complex phase object in the pupil plane, and let us suppose that the statistics of the diffuser are known. Under these conditions that theoretical knowledge allows us to interpret and determine the local phase changes introduced by the object that have taken place by analyzing the modulated fringes in the image plane and comparing them with the situation without the phase object. In summary, our approach provides a tool for determining any local phase change by analyzing the image plane. Finally, it is clear that this approach could be applied in another range of the electromagnetic spectrum where the output of the system is statistically ruled and behaves like speckle structures.

A. Lencina's e-mail address is agl@fisica.ufpb.br.

\section{References}

1. A. Zardecki, Inverse Source Problems, H. P. Baltes, ed. (Springer-Verlag, Berlin, 1985), p. 155.

2. J. C. Dainty, ed., Laser Speckle and Related Phenomena (Springer-Verlag, Berlin, 1975).

3. M. Tebaldi, A. Lencina, and N. Bolognini, Opt. Commun. 202, 257 (2002).

4. L. Angel, M. Tebaldi, M. Trivi, and N. Bolognini, Opt. Lett. 27, 506 (2002).

5. R. P. Khetan and F. P. Chiang, Appl. Opt. 15, 2205 (1976).

6. J. W. Goodman, Introduction to Fourier Optics (McGraw-Hill, New York, 1996), p. 111

7. E. Jakeman, Opt. Eng. 23, 453 (1984).

8. G. Marsaglia and A. Zaman, Ann. Appl. Probab. 1, 462 (1991).

9. K. Uno, J. Uozumi, and T. Asakura, Opt. Commun. 114, 203 (1995)

10. M. Tebaldi, L. Ángel, M. Trivi, and N. Bolognini, J. Opt. Soc. Am. A 20, 116 (2003). 